\title{
La perception des intervenants quant à l'implantation et l'adaptation du programme Intervention comportementale intensive dans le contexte réel d'un établissement québécois Practitioners'perceptions of the implementation and adaptation of the IBI program in the real context of a Quebec rehabilitation center
}

\author{
Valérie Caron, Annie Paquet et Annie Bérubé
}

Volume 48, numéro 2, 2019

URI : https://id.erudit.org/iderudit/1066145ar

DOI : https://doi.org/10.7202/1066145ar

Aller au sommaire du numéro

Éditeur(s)

Revue de Psychoéducation

ISSN

1713-1782 (imprimé)

2371-6053 (numérique)

Découvrir la revue

Citer cet article

Caron, V., Paquet, A. \& Bérubé, A. (2019). La perception des intervenants quant à l'implantation et l'adaptation du programme Intervention comportementale intensive dans le contexte réel d'un établissement québécois. Revue de psychoéducation, 48(2), 303-331. https://doi.org/10.7202/1066145ar

\section{Résumé de l'article}

L'intervention comportementale intensive (ICI) pour les jeunes enfants présentant un trouble du spectre de l'autisme est identifiée comme une pratique basée sur des données probantes (PBDP). Elle a été implantée en 2003 dans les services publics québécois à la suite d'une orientation donnée par le ministère de la Santé et des Services sociaux. Les écrits scientifiques mentionnent clairement les défis des milieux de pratique à implanter, à grande échelle, les PBDP dans les services courants, tout comme la nécessité d'en modifier certains éléments afin de les adapter aux contextes réels d'intervention dans lesquels elles s'implantent. Ce projet vise à documenter l'implantation de l'ICI à partir des représentations des intervenants en ce qui concerne les composantes essentielles de la fidélité de l'ICI, ainsi que des adaptations jugées favorables à sa mise en oeuvre. Ce travail s'inscrit dans le courant des recherches basées sur la pratique en utilisant le savoir des cliniciens comme source d'informations. Les propos de dix intervenants d'un établissement québécois ont été analysés en considérant les cinq dimensions du modèle de Dane et Schneider (1998) pour évaluer la fidélité d'implantation de programmes proposées : l'adhésion, le dosage, la qualité, la participation et la différenciation. Les résultats indiquent que les intervenants ont mis en place l'ICI en regard des caractéristiques essentielles, mais que certaines adaptations ont été effectuées pour favoriser son implantation dans le contexte réel d'un service de réadaptation.
Tous droits réservés ( $\odot$ La Corporation de la Revue Canadienne de Psycho-Éducation, 2019
Ce document est protégé par la loi sur le droit d'auteur. L'utilisation des services d’Érudit (y compris la reproduction) est assujettie à sa politique d'utilisation que vous pouvez consulter en ligne. 


\section{La perception des intervenants quant à l'implantation et l'adaptation du programme Intervention comporte- mentale intensive dans le contexte réel d'un établis- sement québécois}

\section{Practitioners'perceptions of the implementation and adaptation of the IBI program in the real context of a Quebec rehabilitation center}

V. Caron ${ }^{1}$

A. Paquet ${ }^{1}$

A. Bérubé 1

1 Département de psychoéducation, Université du Québec à Montréal

2 Département de psychoéducation, Université du Québec à Trois-Rivières

Correspondance :

Valérie Caron

Université du Québec à TroisRivières, Département de psychoéducation,

3351 Boulevard des Forges, Trois-Rivières, QC, G9A 5H7, valerie.caron1@uqtr.ca

\section{Résumé}

L'intervention comportementale intensive (ICI) pour les jeunes enfants présentant un trouble du spectre de l'autisme est identifiée comme une pratique basée sur des données probantes (PBDP). Elle a été implantée en 2003 dans les services publics québécois à la suite d'une orientation donnée par le ministère de la Santé et des Services sociaux. Les écrits scientifiques mentionnent clairement les défis des milieux de pratique à implanter, à grande échelle, les $P B D P$ dans les services courants, tout comme la nécessité d'en modifier certains éléments afin de les adapter aux contextes réels d'intervention dans lesquels elles s'implantent. Ce projet vise à documenter l'implantation de $\mathrm{I} I \mathrm{Cl}$ à partir des représentations des intervenants en ce qui concerne les composantes essentielles de la fidélité de I'ICl, ainsi que des adaptations jugées favorables à sa mise en œuvre. Ce travail s'inscrit dans le courant des recherches basées sur la pratique en utilisant le savoir des cliniciens comme source d'informations. Les propos de dix intervenants d'un établissement québécois ont été analysés en considérant les cinq dimensions du modèle de Dane et Schneider (1998) pour évaluer la fidélité d'implantation de programmes proposées : l'adhésion, le dosage, la qualité, la participation et la différenciation. Les résultats indiquent que les intervenants ont mis en place I'ICl en regard des caractéristiques essentielles, mais que certaines adaptations ont été effectuées pour favoriser son implantation dans le contexte réel d'un service de réadaptation.

Mots-clés : Intervention comportementale intensive, trouble spectre autisme, fidélité, implantation, adaptations, pratique basée sur des données probantes

\section{Abstract}

Early intensive behavioral intervention for young children with autism spectrum disorders has been identified as an 
evidence-based practice (EBP). As such, the program Intensive Behavioral Intervention (IBI) was implemented in 2003 in Quebec public services following a direction given by the Ministry of Health and Social Services. The scientific literature clearly mentions the challenges of practical settings to implement EBP in mainstream services on a large scale, as well as the need to modify some of their components to adapt them to the actual intervention contexts in which they are implemented. This project aims to document the implementation of the IBI based on practitioners's representations of the essential components of the IBI's fidelity, as well as adaptations deemed favorable to a better fit to their context. This work is in line with practice-based research using clinicians' knowledge as a source of information. The comments of ten practitioners from a Quebec rehabilitation center were analyzed by considering the five dimensions of Dane and Schneider's (1998) model to assess the fidelity of implementation of proposed programs: adherence, dosage, quality, participation and differentiation. The results indicate that the practitioners have implemented the IBI with respect to the essential characteristics, but that some adaptations have been made to facilitate its implementation in the real context of a rehabilitation service.

\section{Keywords: Intensive behavioral intervention, autism spectrum disorders, imple- mentation, fidelity, evidence-based practice, adaptations}

\section{Introduction}

Le trouble du spectre de l'autisme (TSA) est considéré comme un des trouble neurodéveloppemental les plus fréquents (National Autism Center [NAC], 2015). Au Canada, la prévalence de ce diagnostic chez les enfants est estimée à 1 sur 66 (Agence de la Santé Publique du Canada, 2018) et pour la province du Québec, à $1,3 \%$ ou 1 enfant sur 75 en 2015-2016, ce qui correspond à 19425 jeunes de 1 à 17 ans (Institut National de Santé publique du Québec, 2017). Pour les enfants d'âge préscolaire, l'intervention comportementale intensive (ICI) est considérée comme une pratique basée sur des données probantes (PBDP) qui permet des améliorations significatives du fonctionnement des enfants sur le plan adaptatif, de la communication ainsi qu'une diminution de la sévérité des symptômes liés à ce diagnostic, comme le démontrent plusieurs revues systématiques et méta-analyses (Eikeseth, Klintwall, Jahr et Karlsson, 2012; Eldevick et al., 2009; Huffman, Sutcliffe, Tanner et Feldman, 2011; Makrygianni, Gena, Katoudi et Galanis, 2018; Makrygianni et Reed, 2010; National Autism Center, 2009, 2015; Reichow, 2012; Reichow, Barton, Boyd et Hume, 2012; Virués-Ortega, 2010). La somme de connaissances scientifiques au sujet de ce type d'intervention, mais aussi les avis d'experts et les demandes des parents, ont amené certaines provinces canadiennes, dont le Québec, à en favoriser l'accès aux jeunes enfants ayant un TSA (Ministère de la Santé et des Services sociaux [MSSS], 2003).

\section{Les composantes essentielles de l'ICI}

L'intervention comportementale intensive $(\mathrm{ICI})$ regroupe différents modèles globaux (comprehenvise treatment model) basés sur l'analyse appliquée du comportement (AAC) (Odom, Boyd, Hall et Hume, 2010; Odom, Collet-Klingenberg, Rogers et Hatton, 2010). Il y a plusieurs définitions de l'ICI mais un certain consensus sur certaines composantes, soit 1) l'intervention est globale et individualisée ; 2) est offerte durant la période de la petite enfance (dès 3 ans), 3) cible les déficits fondamentaux liés au TSA ; 4) elle est basée sur l'analyse appliquée du comportement 
(AAC) ; 5) elle est enseignée avec un ratio adulte-enfant de 1:1 (intégration graduelle avec pairs) ; 6) est appliquée entre 20 et 40 heures/semaine dans tous les lieux de vie de l'enfant durant 1 à 4 ans et 7 ) elle intègre une composante familiale (formation, supervision, co-intervention) (Reichow, Hume, Barton et Boyd, 2018). Le but de l'ICl est de modifier la trajectoire développementale des jeunes enfants ayant un TSA (Perry, Koudys et Blacklock, 2016). Les études disponibles ont permis d'identifier certaines composantes des programmes d'intervention comportementale intensive précoce qui peuvent influencer les résultats chez les enfants, notamment : l'âge préscolaire, l'application à une intensité de 20 à 40 heures par semaines, la durée d'au moins 12 mois, l'utilisation des stratégies d'enseignement basées sur l'AAC, l'individualisation de l'intervention à partir d'un curriculum regroupant plusieurs domaines de développement dont les domaines déficitaires en TSA et l'utilisation de la collecte de données pour la prise de décision. De plus, l'implication des parents dans l'intervention, l'application dans les différents lieux de vie de l'enfant, l'intégration en milieu ordinaire, la planification de la généralisation et le maintien des acquis, la planification de la transition vers le milieu scolaire ainsi que la formation et la supervision clinique des intervenants (Eikeseth, 2009, 2017; National Research Council [NRC], 2001; Perry (2002); Perry et al. (2016); Rogers et Vismara, 2008). Bien que les recommandations mentionnent une intensité de 20 à 25 heures d'intervention par semaine, la question du dosage ne fait pas totalement consensus et certaines études concluent qu'une intensité moindre peut également entrainer des effets positifs sur le bien-être de la famille (Rivard, Therroux et Mercier (2014). Pour leurs parts, Lindstead et al. (2017) soulignent que c'est plutôt le dosage de l'intervention (la combinaison de l'intensité ou le nombre d'heures avec la durée (en mois) qui affecte les effets et non l'intensité uniquement.

L'efficacité de cette intervention a été abondamment étudiée dans le contexte de la recherche expérimentale (efficacy), mais aussi dans le contexte de services courants (effectiveness) (Bryson, Koegel, Koegel et Openden, 2007; Dionne, Joly, Paquet, Rousseau et Rivard, 2016; Eikeseth, 2009; Eikeseth et al., 2012; Gamache, Joly et Dionne, 2010; Perry et al. 2008; Rivard et al., 2014; Stock, Mirenda et Smith, 2013; Vivanti et al., 2018). Bien que les études menées en contexte de services courants aient obtenus des résultats encourageants quant à l'efficacité de I'ICl, les conditions d'implantation pouvant faire fluctuer l'efficacité ont été peu documentées (Cook et Odom, 2013; Gresham, Gansle et Noell, 1993; Mclntyre, Gresham, DiGenarro et Reed, 2007; Peterson, Horner et Wonderlich, 1982).

\section{L'implantation de I'ICI au Québec}

Au Québec, l'ICl s'inscrit dans une offre de services spécialisés dispensée par les établissements publics (MSSS, 2003). Selon la transformation du réseau québécois, ce sont d'abord les Centres de réadaptation en déficience intellectuelle et trouble envahissant du développement, puis les Centres de réadaptation en déficience intellectuelle et trouble du spectre de l'autisme qui ont mis en œuvre ce programme. Depuis 2015, ce sont les Centres intégrés de santé et de services sociaux (CISSS) ainsi que les Centres intégrés universitaires de santé et de services sociaux (CIUSSS) qui offrent l'ICI dans 22 établissements de santé et services sociaux répartis dans les 18 régions administratives du Québec. Au moment de l'implantation en 2003, certaines lignes directrices ont été proposées aux 
établissements québécois lorsque ceux-ci ont reçu le mandat d'offrir l'ICI (MSSS, 2003). Des précisions concernant la population cible (enfants présentant diagnostic de TSA de 2 à 5 ans) ainsi que l'intensité de l'intervention, soit une vingtaine d'heures d'intervention par semaine par enfant, ont été fournies en soutien à la mise en œuvre de ce programme (MSSS, 2003). Concernant les modalités d'intervention, le MSSS (2003) préconisait que l'ICl intègre « les approches adaptées aux troubles envahissants du développement, notamment, l'approche Lovaas, l'approche TEACCH (Treatment and Education of Autistic and related Communication handicapped CHildren) et le système de communication "Picture Exchange Communication System (PECS) » (MSSS, 2003, p. 38). En 2008, un guide de pratique basé sur des savoirs d'experts a été publié par la Fédération québécoise des Centres de réadaptation en déficience intellectuelle et trouble envahissant du développement (FQCRDITED) en soutien à l'implantation de ce programme dans les établissements québécois (FQCRDITED, 2008). En 2014, I'Institut national d'excellence en santé et en services sociaux (INESSS, 2014a; 2014b) a préconisé que l'ICI soit l'intervention privilégiée pour les enfants d'âge préscolaire présentant un TSA, compte tenu de ses effets sur le fonctionnement cognitif, le langage et le comportement adaptatif, et ce, à raison d'une intensité minimale de 20 heures par semaine. En 2017, le MSSS a publié un plan d'action sur les TSA regroupant plusieurs objectifs dont « l'amélioration de l'accès et de la qualité de l'application de I'ICI dans les différents milieux de vie de l'enfant; la documentation d'autres approches basées sur les meilleures pratiques et la diversification de l'offre de services spécialisés selon les profils et les besoins " (MSSS, 2017, p. 11). Pour ce faire, le MSSS préconise de « définir des lignes directrices ministérielles pour baliser l'application de l'ICl et d'établir des balises pour l'utilisation d'approches d'intervention de réadaptation autre que l'ICI » (MSSS, 2017, p. 12).

Entre temps, les établissements concernés par la mise en œuvre de l'ICl ont été amenés à faire des choix relativement à la façon d'implanter I' $\mathrm{ICl}$ dans leurs organisations respectives (Dionne et al., 2016). Malgré des efforts importants de leurs parts pour mettre en place une intervention respectant les principales composantes de l'ICl et les lignes directrices proposées, certains défis d'implantation ont été soulevés et, en conséquence, la facon d'offrir l'intervention s'est articulée différemment d'un centre à l'autre, voire au sein d'un même établissement (Dionne et al., 2016; Gamache et al., 2010). En effet, les études québécoises ont mis en lumière certains défis liés à l'implantation de l'ICI, notamment, la grande diversité des pratiques, la variabilité dans les modèles de supervision clinique, le manque de documentation spécifique, de guide ou de matériel d'intervention (surtout francophone), le manque de ressources humaines et le dosage de l'intervention inférieur au dosage de la vingtaine d'heures préconisée par le MSSS (Dionne et al., 2016; Gamache et al., 2010; Paquet, Dionne, Joly, Rousseau, Rivard, 2017; Rivard et al., 2014). À la suite de ces constats, les chercheurs québécois recommandent de fournir une documentation officielle sur ce programme et de poursuivre les efforts visant à soutenir la qualité de l'implantation de l'ICI (Dionne et al., 2016; Gamache et al., 2010; Paquet et al., 2017; Rivard et al., 2014). 


\section{La mise en œuvre des PBDP dans les services courants}

Le contexte du Québec ne représente pas une exception mais plutôt un constat généralisé. En effet, les écrits scientifiques soulignent que l'existence des PBDP n'est pas suffisante pour assurer leur implantation dans les services (Aarons et al., 2012; Cook et Odom, 2013). En ce sens, la combinaison d'une PBDP avec une mise en œuvre efficace est nécessaire pour engendrer les effets escomptés des interventions (Fixsen, Blase, Metz et Van Dyke, 2013). Bien que les défis d'implantation des PBDP soient bien documentés, ils demeurent d'actualité, notamment dans le champ des interventions en TSA (Cook et Odom, 2013). Pour réduire cet écart, la science de l'implantation (en anglais, implementation science) a recours à l'évaluation de l'implantation pour étudier les contextes dans lesquels les PBDP sont mises en œuvre sur le terrain (Fixsen, Naoom, Blasé, Friedman et Wallace, 2005). Celle-ci n'étudie pas les effets des PBDP, mais plutôt leur mise en œuvre dans divers contextes de services courants (real world context). Elle s'intéresse aux étapes de la mise en place d'une PBDP dans la pratique : le choix d'une PBDP, son adoption par les milieux de pratique ou pré-implantation, son implantation, l'évaluation de son implantation en contexte réel (Fixsen et al., 2005; Metz, Halle, Bartley et Blasberg, 2013). Pour ce faire, la science de l'implantation recommande d'évaluer la fidélité de l'intervention mais aussi de documenter les adaptations effectuées en regard de l'intervention prévue initialement (Chen, 2015; Dunsenbury, Brannigan, Hansen, Walsh et Falco, 2005; Durlak et Dupré, 2008; Pérez et al., 2016).

\section{Adaptation c. Fidélité des PBDP}

La fidélité de l'implantation, aussi connue sous le terme de l'intégrité de l'intervention (integrity) est définie par le degré de conformité de la mise en place d'une intervention en regard de ce qui était prévu à la base (Dane et Schneider, 1998; Moncher et Prinz, 1991; Hagermoser Sanetti et Kratchowill, 2009). Bien que les définitions des termes de la fidélité et de l'adaptation (reinvention, modification) des PBDP semblent s'opposer, certains auteurs soulignent que les deux sont complémentaires et qu'ils devraient coexister dans le processus d'implantation dans les services courants (Aarons et al., 2012; Chen, 2015; Durlak et Dupré, 2008; Dunsenbury et al., 2005; Pérez et al., 2016). Les adaptations aux PBDP sont considérées fréquentes et bénéfiques lorsqu'elles permettent une meilleure adéquation entre les contextes d'origine dans lesquels les pratiques ont été évaluées et les contextes de services courants dans lesquels elles s'implantent par la suite (Aarons et Palinkas, 2007, Aarons et al., 2012; Durlak et Dupré, 2008; Escoffery et al, 2018). Pour cela, différents types d'adaptations peuvent être réalisés par les milieux, notamment, la modification du contenu (ajout et retrait de certains éléments prévus), le matériel pédagogique, la durée, la structure des activités, l'intégration de nouvelles approches d'intervention ou la modification pour un public-cible différent (Escoffery et al., 2018). Les principales raisons qui sous-tendent ces adaptations sont, le besoin d'adaptation culturelle, une nouvelle population cible ou un contexte d'implantation différent (Escoffery et al., 2018). 
Un mauvais équilibre entre la fidélité et l'adaptation peut engendrer des effets moindres que ceux documentés dans le cadre des études évaluatives (Aarons et al., 2012). Pour éviter ce déséquilibre, la notion de composantes principales (core components ou active ingredients), définies par les composantes jugées nécessaires pour atteindre les effets escomptés, s'est imposée (Aarons et al., 2012). Ces composantes sont associées aux éléments centraux d'un intervention qui définissent fondamentalement sa nature et ont une influence sur les effets, tels que déterminés par la théorie du programme, le modèle logique ou les preuves scientifiques (Backer, 2001; Lee, Altschul et Mowbray, 2008). Comme ces composantes principales sont celles qui expliquent les effets, elles ne devraient pas être modifiées ou « adaptées ». En contrepartie, les composantes non principales (dites secondaires) peuvent être adaptées aux différents contextes et particularités organisationnelles sans en affecter négativement les effets (Aarons et al., 2012; Michie, Fixsen, Grimshaw et Eccles, 2009). Dans tous les cas de figure, les adaptations effectuées aux composantes principales sont susceptibles d'affecter les effets de l'intervention (Aarons et al., 2012; Castro, Barrera et Martinez, 2004; Fixsen et al., 2013; Hansen et al., 2013). Pour trouver l'équilibre entre la fidélité et l'adaptation des interventions dans la pratique, les écrits scientifiques suggèrent d'évaluer la fidélité de l'implantation des PBDP dans le contexte réel et de documenter les adaptations effectuées par les milieux de façon à pouvoir comprendre et contextualiser l'efficacité (Aarons et al., 2012; Chen, 2015; Dane et Schneider, 1998; Durlak et Dupré, 2008). En ce sens, l'évaluation de la fidélité est donc essentielle pour décrire l'intervention mise en œuvre dans la pratique, mais aussi pour interpréter adéquatement les résultats lors de l'évaluation des effets (Aarons et Palinkas, 2007; Dane et Schneider, 1998, Dunsenbury et al., 2003; Durlak et Dupré, 2008; Leclair, Paquette et Letarte, 2017).

\section{L’évaluation de la fidélité}

Bien que son importance soit reconnue depuis plusieurs années, l'évaluation de la fidélité est effectuée dans une minorité d'études sur l'ICl et elle est rarement considérée dans l'analyse des effets de l'ICI (Caron, Bérubé et Paquet, 2017; Cook et Odom, 2013; Odom et al., 2010; Odom, Cox et Brock, 2013). En effet, les données sur l'implantation sont souvent décrites de façon partielle, notamment, par la mention du dosage prescrit, du curriculum utilisé et, dans certains cas, par l'évaluation du dosage offert, mais les informations sur l'implantation sont souvent partielles et incomplètes (Caron et al., 2017). Pourtant, différents modèles d'évaluation existent, suggérant des dimensions à considérer lors de l'évaluation de la fidélité d'implantation d'un programme. Celui de Dane et Schneider (1998) a été repris par plusieurs auteurs dans le cadre de l'évaluation de l'implantation de programmes d'intervention psychosociale offerts dans les services publics (Bérubé et al., 2014; Dunsenbury et al., 2003; Dunsenbury et al., 2005; Paquette, Joly et Tourigny, 2010). Pour ce faire, cinq dimensions de la fidélité de l'implantation sont proposées par ce modèle : 1) l'adhésion ou la conformité de l'intervention par rapport au programme prévu; 2) le dosage ou le degré d'exposition des participants au programme; 3) la qualité de l'intervention, par exemple la qualité de la relation entre l'intervenant et l'enfant; 4) la participation, à savoir le degré d'engagement des participants dans le programme et 5) la différenciation, soit la valeur ajoutée du programme par rapport à d'autres interventions offertes pour le même public cible. 
Ce modèle permet de recueillir une multitude d'informations sur un programme, mais aussi de nuancer les effets d'une intervention à la lumière des informations recueillies (Dane et Schneider, 1998; Dunsenbury, Brannigan, Falco et Hansen, 2003).

Dans le contexte québécois, les lignes directrices offertes par le MSSS pour la mise en œuvre de l'ICl semblent avoir laissé une certaine marge de manœuvre aux établissements en ce qui concerne l'implantation de l'ICI. En conséquence, les choix des établissements québécois sont variés et l'implantation de ce programme semble avoir fait l'objet de plusieurs adaptations locales (Dionne et al., 2016; Gamache et al., 2010; Paquet et al., 2017). Bien que celles-ci soient certainement jugées nécessaires par les différents établissements, elles n'ont pas fait l'objet d'évaluation tel que le recommandent les écrits scientifiques et sont donc peu documentées. Le MSSS (2017), tout comme les chercheurs québécois (Dionne et al., 2016), recommandent d'améliorer l'application de l'ICl ainsi que le soutien à son implantation, en fournissant, notamment une documentation officielle ainsi qu'une diversification de l'offre de services spécialisés selon les profils et les besoins.

Considérant l'état d'avancement des connaissances à la fois dans le domaine de I'ICl et relativement aux meilleures pratiques d'implantation des programmes exposés précédemment, il apparaît tout à fait indiqué de tenter de mieux comprendre comment l'implantation du programme $\mathrm{ICl}$ est perçue par les intervenants sur le terrain. Pour ce faire, cette étude s'inspire des recherches basées sur la pratique en utilisant le savoir des cliniciens comme source d'information (Crooke et Olswang, 2015; Olswang et Prelock, 2015). Elle a comme objectif de documenter la perception des intervenants quant à l'implantation de l'ICl dans un établissement québécois. Elle vise à décrire leur point de vue sur les composantes essentielles de la fidélité de l'implantation de l'ICI ainsi que les adaptations jugées favorables. Cette recherche a été approuvée par le comité éthique de l'Université du Québec à Trois-Rivières ainsi que le comité d'éthique de l'établissement concerné.

\section{Méthode}

\section{Participants}

L'étude se penche sur la perception qu'ont les intervenants de l'implantation de l'ICl en prenant comme objet d'étude un établissement du réseau de la santé et des services sociaux québécois. Au moment de cette recherche, le service offrant le programme ICl était composé de 16 intervenants et accueillait 20 enfants ayant un TSA. Tous les intervenants ont été invités par écrit à participer à des entrevues, à la suite d'une présentation du projet. Dix intervenants de ce service $(62,5 \%)$ ont accepté de participer au projet et ont été interrogés (sept intervenants, deux superviseurs et une professionnelle) (un homme et neuf femmes). Parmi ceux-ci, tous étaient embauchés sous le titre d'emploi de conseiller en enfance inadaptée sauf pour la professionnelle (ergothérapeute). Ils ont des formations initiales diverses ainsi qu'un nombre d'années d'expérience moyen de 7,2 années (écart type de 5,03) (Tableau 1). Parmi l'échantillon, un seul intervenant a complété une formation de deuxième cycle spécialisée en TSA. 
Tableau 1. Description des intervenants

\begin{tabular}{lcl}
\hline \multicolumn{2}{c}{ Années d'expériences } & \multicolumn{1}{c}{ Formation } \\
\hline P1 & 15 & Baccalauréat en psychoéducation et DESS en supervision \\
P2 & 4 & Maîtrise en psychoéducation \\
P3 & 4 & Maîtrise en psychoéducation \\
P4 & 9 & Baccalauréat en psychoéducation et maîtrise en cours \\
P5 & 10 & Baccalauréat en psychologie \\
P6 & 2 & Maîtrise en psychoéducation \\
P7 & 10 & Baccalauréat adaptation scolaire \\
P8 & 14 & Maîtrise en psychoéducation \\
P9 & 2 & Baccalauréat en ergothérapie \\
P10 & 2 & Maîtrise en psychoéducation \\
\hline
\end{tabular}

Le choix de recruter des participants d'un seul établissement a été fait afin d'avoir une vision en profondeur du point de vue des intervenants qui travaillent au sein du même établissement. II n'est pas rare que le nombre de participants dans le cadre d'études qualitatives en sciences sociales au Québec soit en deçà de 20 (Royer, Baribeau et Duschesne, 2009). Le taux de participation (62,5\% des membres de l'équipe), de même que la diversité des profils des répondants suggèrent une certaine représentativité.

\section{Procédure}

Des entrevues semi-dirigées, d'une durée variant entre une heure et une heure et demie, ont été effectuées auprès de chacun des intervenants par la première auteure. En ce qui concerne la durée des entretiens, elle est généralement entre 60 et 90 minutes (Royer et al. 2009). L'utilisation de mesures auto-rapportées, recueillies sous forme d'entretien, sont régulièrement utilisées pour documenter l'implantation d'une intervention en contexte de services courants (Breinstein et al. 2010). Elles sont considérées comme une source d'information pertinente pour documenter la mise en œuvre et l'adhésion des participants au protocole d'intervention prévu (Lee et al. 2008). Les entrevues ont été menées entre les mois de mai et juillet 2014 et ont été enregistrées, retranscrites et codées.

\section{Instrument}

Un guide d'entrevue dirigée a été élaboré par l'équipe de recherche en prenant appui sur le modèle de Dane et Schneider (1998) (Annexe A). Les questions portaient sur la perception des intervenants du programme et de son application dans leur établissement. Les entrevues ont permis d'aborder les perceptions des intervenants quant aux cinq dimensions du modèle, à savoir: 1) l'adhésion (le degré de conformité de l'intervention, les modifications apportées, le modèle d'intervention préconisé, les documents de référence, les éléments faciles à mettre en place et les obstacles rencontrés); 2) le dosage (le nombre d'heures et les lieux d'intervention); 3) la qualité (la relation intervenant-enfant et les mécanismes d'assurance qualité 
mis en place); 4) la participation (les acteurs impliqués, le niveau d'engagement des participants) et 5) la différenciation (la valeur ajoutée d'une intervention par rapport à une autre, des programmes complémentaires). De plus, trois questions concernant la formation et les expériences professionnelles des participants précédaient l'entretien, soit la formation initiale, la formation continue et le nombre d'années d'expérience en ICl.

\section{Stratégies d'analyse}

Une analyse thématique des données a été réalisée (Paillé et Mucchielli, 2012). Elle correspond à la « transposition d'un corpus donné en un certain nombre de thèmes représentatifs du contenu analysé et ce, en rapport avec l'orientation de la recherche » (Paillé et Mucchielli, 2012, p.124). Le logiciel N-Vivo 11 a été utilisé afin de faciliter la gestion du matériel recueilli. Ainsi, une transcription des verbatim, suivie d'une première étape de lecture latente, a permis de se familiariser avec le contenu. Le contenu des entrevues a d'abord été regroupé en fonction de grandes rubriques correspondant aux cinq dimensions du modèle d'évaluation retenu, soit le modèle de Dane et Schneider (1998). Au sein de chacune des rubriques, un processus de thématisation en continu a été utilisé, c'est-à-dire un travail d'attribution de thèmes et, simultanément, de construction de l'arbre thématique. Ainsi, des analyses, suivies d'échanges et de discussions avec les deuxième et troisième auteures ont permis de valider et d'affiner les thèmes, ainsi que l'arbre thématique, tout au long du processus.

\section{Résultats}

Les résultats sont présentés selon les cinq dimensions du modèle de Dane et Schneider (1998). L'analyse porte donc sur la perception qu'ont les intervenants du programme, tel qu'il est implanté en regard de 1) l'adhésion; 2) du dosage; 3) de la qualité; 4) de la participation et 5) de la différenciation. Pour chacune des dimensions, les informations ont été regroupées de façon à documenter la perception des intervenants quant aux composantes principales de $\mathrm{I}^{\prime} \mathrm{ICl}$ et les adaptations qu'ils jugent utiles ou nécessaires.

\section{L'adhésion}

La dimension de l'adhésion concerne la conformité de l'intervention par rapport au programme prévu. En ce qui concerne le modèle d'intervention, les ouvrages de référence et les stratégies d'intervention, les intervenants évoquent une certaine évolution de leurs pratiques d'intervention en $\mathrm{ICI}$ depuis le début de l'implantation du programme dans leur établissement en 2003. Actuellement, les intervenants estiment que l'ICl, telle que mise en place dans leur établissement, est basée sur l'AAC, mais plus spécifiquement sur un modèle d'intervention de l'AAC qui intègre l'approche fonctionnelle des comportements verbaux proposée par Skinner (1957), soit le modèle ABA-VB (Applied Behavior Analysis-Verbal Behavior) (Sundberg, 2008). Les intervenants décrivent le modèle ABA-VB comme étant conçu pour motiver les enfants à apprendre à communiquer et à développer une communication fonctionnelle. Ils soulignent que ce choix permet une harmonisation de leurs pratiques en leur permettant de s'appuyer sur un modèle de référence 


\section{2}

commun et complet. Le modèle ABA-VB propose une intervention centrée sur le développement du langage (à partir de l'enseignement des opérants verbaux de la communication, mais leur modèle intègre également des objectifs d'intervention qui concernent des habiletés fonctionnelles et les barrières à l'apprentissage (comportements-défis).

Ils considèrent que les composantes essentielles de leur programme ICI sont: 1) l'évaluation de l'enfant, 2) l'évaluation des renforçateurs, 3) la rédaction d'objectifs d'intervention, 4) la planification des stratégies spécifiques d'intervention, 5) le recueil de données et la révision des objectifs d'intervention et 6) la préparation de la transition vers le milieu scolaire.

1.1 L'évaluation de l'enfant est réalisée à l'aide du curriculum VB-MAPP (Verbal Behavior - Milestones Assessment and Placement Program) ${ }^{1}$ (Sundberg, 2008). Selon les intervenants, cette évaluation permet de fournir un portrait de l'enfant et d'identifier des cibles d'intervention de façon très précise en plus d'harmoniser les pratiques d'évaluation et de planification de l'intervention dans leur établissement. II permet également de suivre l'évolution de l'enfant et d'ajuster les cibles d'intervention durant le programme. «Avec le VB-MAPP, je trouve qu'on voit beaucoup plus l'évolution de l'enfant [...] Puis c'est facile d'aller chercher les objectifs et aussi d'atteindre des buts avec l'enfant. [...] Oui, avec le VB je vois plus de sens. ॥ (P7). L'outil permet également d'évaluer les barrières à l'apprentissage (section de l'outil VB-MAPP) et de réaliser une analyse fonctionnelle des comportements-défis lorsqu'ils sont présents. Les intervenants soulignent que cette analyse permet de rédiger le plan d'intervention visant l'enseignement des comportements alternatifs.

II faut lui donner les bons moyens [...] Si l'enfant frappe et fait des crises, il n'est pas bien. Donc, je veux lui donner une meilleure carte [...] Tu sais, nous autres c'est ce qu'on fait. On fait de l'extinction sur ses mauvaises cartes et on lui en donne des meilleures [...] Oui, chacune des cartes a été analysée et en plus, on a montré aux parents comment jouer. (P1) (superviseur)

En complément à cette évaluation initiale, les intervenants conçoivent que d'autres outils d'évaluation de développement standardisés ainsi que différentes observations ou grilles d'évaluation tirées de différents curriculums d'intervention (par exemple, Maurice et al., 1996; Lovaas, 2003) peuvent être combinés, de façon optionnelle, pour préciser le profil de l'enfant, mais l'outil d'évaluation privilégié pour tous les enfants est le VB-MAPP.

1.2 L'évaluation des renforçateurs. Les intervenants conçoivent que l'évaluation des renforçateurs est une composante essentielle de l'ICl. Selon eux, elle devrait être réalisée au début de l'intervention, mais également à chacune des

1. L'évaluation est divisée en plusieurs domaines d'évaluation correspondant aux comportementaux verbaux définis par Skinner (1957) divisés en trois niveaux d'âge (018 mois; 18-30 mois; 30-48 mois) et sous-divisés en 170 jalons (milestones) qui sont des habiletés représentatives du développement du langage. 
séances afin de pouvoir utiliser les intérêts de l'enfant et susciter sa motivation pour les apprentissages. Ils mentionnent l'importance d'utiliser et de varier les différents types de renforçateurs primaires (p. ex., alimentaires, jouets) et secondaires (p. ex., sociaux, systèmes de jetons) afin d'éviter la saturation.

1.3 La rédaction des objectifs d'intervention. Les intervenants soulignent que la rédaction des objectifs d'intervention constitue également une composante essentielle du programme $\mathrm{ICl}$, car elle permet d'identifier les objectifs d'intervention en combinaison avec les priorités des parents. Les objectifs sont basés sur l'évaluation VB-MAPP qui permet la planification et le suivi des objectifs d'intervention. "Nous autres, on a un programme de 18 pages avec du détail, c'est tout spécifique, micro-gradué et très précis »(P4). Ils soulignent que les objectifs devraient être rédigés de façon opérationnelle et permettre la planification des séances d'intervention. "Avoir un objectif de base, précis, observable et mesurable et de bâtir les activités autour de cet objectif et non le contraire. ॥ (P2). Les intervenants soulignent que le modèle actuel implanté à partir de l'ABA-VB les amène à cibler des objectifs plus fonctionnels pour les enfants qu'avec les curriculums utilisés lors des premières années d'implantation.

C'est le fun de travailler le rouge, le jaune et le vert, mais est-ce que ça va l'aider dans la vie s'il n'est pas capable de suivre maman à l'épicerie, s'il n'est pas capable de s'asseoir dans l'auto sans se détacher. (P3)

1.4 La planification des stratégies d'intervention. Les intervenants soulignent qu'il est nécessaire d'utiliser une variété de stratégies d'intervention basées sur l'AAC. Ils considèrent que celles-ci devraient être individualisées à chaque enfant et adaptées tout au long du programme, selon l'évolution de chacun. Ainsi, ils considèrent désormais que la planification des séances devrait permettre de multiplier les occasions d'apprentissage en insérant les objectifs d'intervention dans la vie quotidienne, tout en prenant appui sur la motivation de l'enfant et la routine de la famille. Cela permet aux enfants de faire des apprentissages fonctionnels et d'utiliser leurs compétences au quotidien. Ainsi, les intervenants privilégient l'enseignement dans l'environnement naturel afin de permettre de généraliser les objectifs dans les différents milieux de vie. "Cela augmente aussi le potentiel d'apprentissage étant donné qu'on n'est pas tout le temps tout seul justement à la table, qu'on utilise toutes les opportunités de l'environnement. » (P9)

L'enseignement par essais distincts (angl. Discrete Trial Teaching) est une stratégie qui devrait être utilisée en ICl selon les intervenants. Ils soulignent que cette stratégie était majoritairement utilisée lors des premières années de l'ICI, mais ils estiment qu'elle devrait être combinée, autant que possible, avec l'enseignement dans l'environnement naturel. C'est d'ailleurs le recours au modèle ABA-VB qui a entrainé cette modification dans le choix des stratégies d'enseignement. Ils considèrent désormais l'importance de combiner les stratégies d'intervention et d'avoir recours à l'enseignement dans l'environnement naturel pour développer la communication fonctionnelle de l'enfant. En ce sens, les intervenants mentionnent qu'ils planifient leurs séances d'intervention judicieusement en intégrant des occasions de demandes en combinaison avec les intérêts et les acquis des enfants 
dans les séances d'apprentissage de façon à rendre les séances plus motivantes. « J'essaie d'avoir plusieurs petits moments de travail en apprentissage parce que je suis la disponibilité de l'enfant. Si c'est 25 minutes, on va alterner avec autre chose et on va retourner faire un autre 25 minutes. " (P10)

L'enseignement d'un moyen de communication constitue une composante principale du programme ICI lorsque les enfants présentent des déficits en communication car il permet de prévenir le développement de comportements défis.

«Le PECS ${ }^{2}$ ça prend du temps à faire, mais s'il en a besoin, même si c'est juste pour 3 mois, ce n'est pas grave, on le met en place puis, ça va prendre le temps que ça va prendre [...] On évite bien des crises. ॥ (P7)

L'enseignement d'habiletés sociales peut aussi faire partie intégrante des éléments à travailler en $\mathrm{ICl}$ et représente également une composante essentielle du programme selon les intervenants. Ils soulignent toutefois que ces compétences devraient être enseignées dans la mesure où l'enfant possède les prérequis dans les résultats de l'évaluation du domaine social de l'outil VB-MAPP. Ils proposent de travailler ces compétences en dyade (deux enfants) ou en groupe dans les milieux réguliers tels que les milieux de garde ou les milieux scolaires et préscolaires. Ceci représente une adaptation effectuée par rapport au programme initial.

L'après-midi, c'est pour la généralisation et l'autonomie et aller au parc et faire le social. Par exemple, les habiletés sociales, c'est cela qui ressemble beaucoup à du natural environnemental teaching. (P1) (superviseur)

Les scénarios sociaux ${ }^{3}$ constituent une autre stratégie d'enseignement couramment utilisée en $\mathrm{ICl}$ pour les enfants qui possèdent un niveau de fonctionnement correspondant au niveau 2 et 3 selon l'outil VB-MAPP.

Les scénarios, c'est plus dans la vie de tous les jours. Moi je l'utilise plus avec les enfants qui sont très forts. Comme j'ai un petit, on est à l'école, la cloche a sonné, il ne rentrait pas parce qu'il jouait. Donc c'est juste lui montrer quand la cloche sonne, je prends mon rang, je rentre. (P3)

Les intervenants mentionnent que la structure de l'environnement (référant le plus souvent à TEACCH) ainsi que les supports visuels sont très utilisés pour donner des repères spatiaux ou visuels aux enfants et les rendre plus autonomes.

2. Le PECS réfère au système de communication par échanges d'images (Picture Exchange Communication System) créé par Bondy et Frost, 1994.

3. Les scénarios sociaux ont été créés par Gray (1994). II s'agit d'un outil visuel présenté sous la forme de courtes histoires dont le but est de décrire une situation sociale et d'enseigner les comportements attendus. 
Moi je lui montre la séquence, je ne dis pas un mot, je mets le timetimer (horloge visuelle), il lit ça, il regarde le time-timer et ça fait " bip », il s'en va, il s'enligne, puis il fait la transition. [...] II a juste besoin de quelque chose qui est clair, qui sonne et qui lui dit que je suis rendu à autre chose. (P8)

En ce qui concerne les adaptations effectuées, les intervenants rapportent l'utilisation des appareils numériques à plusieurs fins dans l'intervention, notamment, pour filmer des séances d'intervention ou certains comportements de l'enfant pour lesquels ils souhaitent avoir différents avis pour l'intervention. " Je prenais des vidéos pour documenter une situation, par exemple, avec le pédopsychiatre, le médecin, le pédiatre [...] Pour le montrer, pour qu'ils comprennent un peu la situation. » (P6)

De plus, ils rapportent qu'ils utilisent également les appareils numériques pour préparer des séquences visuelles ou pour enseigner certaines habiletés par modelage vidéo. Par contre, ils mentionnent que l'enseignement de la communication par tablettes numériques est peu utilisé, car ce moyen peut être plus contraignant que l'apprentissage de gestes, de signes ou de pictogrammes.

C'est une intervention qui est vraiment plus rare parce que ce n'est pas ce qu'on vise. Ce n'est pas naturel d'avoir un appareil de communication pour un enfant [...] Je trouve que le Ipad pourrait être plus utilisé à des fins éducatives et il est un petit peu malheureusement plus utilisé en renforçateurs. (P2)

1.5 Le recueil des données et la révision des objectifs d'intervention. Les intervenants soulignent que le recueil des données tout comme la révision des objectifs constituent des composantes essentielles au programme $\mathrm{ICl}$ car ils permettent de recueillir des informations sur l'évolution des objectifs d'intervention par le biais d'une prise de données réalisée à chaque séance d'intervention. Ainsi, ils soulignent que ce recueil peut s'effectuer de différentes manières mais qu'ils ont, pour leur part, privilégié la méthode de la cotation par échantillonnage à froid (cold probe) lors du premier essai de la séance d'intervention de l'enfant, car il la juge plus pertinente que la cotation de chaque essai, tout en leur permettant d'assurer un suivi rigoureux des cibles d'apprentissage.

Le cold probe c'est le matin-là ou en début de séance. Tu testes tes cibles, tu as entre dix et 20 cibles dans ta journée peu importe que cela soit des mots, des activités en lien avec tes objectifs spécifiques [...] Durant la thérapie, je vais continuer d'enseigner, par exemple, le chapeau, je vais le travailler plusieurs fois, mais je ne le coterai plus. (P1) (superviseur)

En ce qui concerne la révision des objectifs d'intervention, les intervenants soulignent que celle-ci devrait être effectuée régulièrement tout comme la mise à jour de l'outil d'évaluation de référence VB-MAPP. «Le VB-MAPP, on le met à jour au minimum aux trois mois ». (P5) 


\subsection{La préparation de la transition vers le milieu scolaire}

Les intervenants soulignent que l'une des composantes essentielles du programme $\mathrm{ICl}$ est la préparation des enfants à la transition vers le milieu scolaire. Pour cela, ils effectuent un bilan de l'enfant visant à décrire le fonctionnement actuel tout comme les stratégies d'intervention à privilégier afin d'assurer une transition harmonieuse entre les services. Pour préparer cette transition, les intervenants rapportent que leur outil principal de référence est le portfolio de l'enfant ainsi que l'Évaluation des possibilités d'orientation du VB-MAPP.

« En fait, il y a un outil de transition qui vient avec le VB-MAPP [...] Donc, c'est comme pour faire ton portrait à la fin de ton $\mathrm{ICl}$, tu peux aller coter tous tes jalons. » (P5). De plus, cette transition s'effectue à partir du transfert d'informations entre les acteurs du milieu scolaire et les intervenants du programme ICl.

Je donne aux enseignants tout le matériel que j'ai utilisé dans l'année précédente. Ils peuvent s'en faire des photocopies [...] Des scénarios sociaux, des bandes illustrées, des routines, des stratégies gagnantes, on fait tout le temps le petit rapport. On essaie que ça ressemble le plus possible à ce qu'ils vont vivre à l'école. (P8)

\section{Le dosage}

Cette dimension concerne le degré d'exposition au programme (Dane et Schneider, 1998). Malgré une orientation ministérielle unique d'une vingtaine d'heures par semaine et la reconnaissance dans la littérature scientifique qu'une intensité soit nécessaire, les intervenants considèrent que le dosage actuel (entre 14 et 16 heures) répond aux besoins. "En moyenne, ça dépend, c'est 16 heures pour les équipes à trois puis 14 pour les équipes à 4. [...]. Moi, je trouve que 14 à 16 heures, c'est très bien. » (P5). Les intervenants soulignent que le dosage pour chaque enfant est offert dans divers milieux de vie tel que le domicile familial, le milieu de garde, le centre de réadaptation et l'école préscolaire. Les intervenants soulignent que le dosage unique préconisé par le MSSS n'est pas favorable pour tous les enfants et que, dans certains cas, celui-ci doit être adapté en fonction des besoins et de la disponibilité des enfants en cours de programme. Ils considèrent que le dosage optimal de l'ICl devrait être un dosage souple qui leur permet de bien répondre aux besoins des enfants selon les situations.

Tu sais, avec un petit coco de 2 ans et demi, 3 ans, 3 ans et demi, tu sais, 16-20 heures, cela n'a pas de bon sens. Physiquement, un enfant n'est pas capable de tenir cela [...]. II s'endort à côté de moi. II n'en peut plus. (P3)

Ils considèrent que le dosage optimal est celui qui devrait permettre d'intervenir dans toutes les sphères du développement de l'enfant et ce, dans les différents milieux de vie tels que le domicile familial, le milieu de garde ou l'école maternelle. 
Parce qu'on peut aller vraiment en profondeur « [...] Au niveau des parents, c'est rassurant d'avoir quelqu'un aussi. II ne dort pas, le soir, moi je vais venir faire des soirs chez vous, je vais t'aider avec la routine du dodo. Ça fait vraiment partie du service ICl. (P4)

\section{La qualité}

La dimension de la qualité concerne deux aspects, soit la qualité de la relation entre l'intervenant et l'enfant et le soutien organisationnel permettant d'assurer la qualité de l'intervention au sein du programme. Ces deux aspects sont considérés globalement par les intervenants comme des composantes essentielles, mais dont les stratégies à mettre en place devraient être ajustées afin de s'adapter aux besoins de chacun.

3.1 La qualité de la relation avec l'enfant. Les intervenants soulignent que la qualité de la relation entre l'enfant et l'intervenant (définie comme la notion de pairing dans le modèle $A B A-V B$ ) constitue une composante essentielle du programme ICl puisqu'elle permet, par la suite, l'application du programme d'une façon plus fluide. Les intervenants soulignent également l'importance de la mise en place du contrôle instructionnel auprès des enfants. " Au début, on est plus dans le plaisir. On va en demander moins à l'enfant, on donne plus de renforcement. " (P6). Ils le décrivent essentiel au démarrage de l'apprentissage des objectifs d'intervention mais également tout au long du programme $\mathrm{ICl}$, car il vise à motiver l'enfant, à rendre intéressants les moments partagés avec l'intervenant et les activités proposées pour ensuite passer aux apprentissages plus formels.

Le contrôle instructionnel, c'est de faire comprendre à l'enfant pourquoi il doit travailler. De lui faire comprendre qu'il y a un échange, que si je vais te demander quelque chose, ce ne sera pas toujours plate et que des fois, ça ne te tente pas de travailler mais tu vas avoir quelque chose au bout [...]. Dans le fond, c'est par ça qu'on arrive à tout instaurer l'apprentissage et si tu n'as pas ça, tu ne peux pas faire grand-chose avec l'enfant parce que c'est lui qui a le contrôle, c'est pas toi. Donc ça c'est important. (P6)

3.2 Le soutien organisationnel pour assurer la qualité. Les intervenants considèrent que la qualité de I'ICI repose sur plusieurs piliers organisationnels. D'abord, ils estiment que la formation initiale est primordiale pour permettre à tous d'acquérir les connaissances de base avant de démarrer au programme $\mathrm{ICl}$ et pour faciliter l'adhésion et la compréhension du programme. Les intervenants soulignent que le travail d'équipe interdisciplinaire constitue une composante essentielle du programme $\mathrm{ICl}$, ce qui leur permet de partager les points de vue cliniques concernant les stratégies d'intervention. "Le travail interdisciplinaire nous aide beaucoup. " (P5). De plus, ils considèrent que le fait de travailler en équipe auprès de l'enfant permet de généraliser les objectifs. «Travailler aussi en trio. Ça apporte beaucoup justement au niveau du recul puis le fait de se remettre en question. On n'est pas toujours tous d'accord sur tout. » (P5) 
Les intervenants soulignent que la supervision clinique constitue une autre composante essentielle du programme $\mathrm{ICl}$ car elle permet un soutien clinique ainsi que des conseils provenant d'une équipe clinique aux différentes étapes du programme. Pour ce faire, une équipe de superviseurs composée de 5 professionnels a été mise en place (psychoéducateurs, orthophonistes et ergothérapeutes) pour offrir du soutien sur demande à l'équipe d'intervenants qui appliquent l'ICl.

Tu sais, pour avoir un jugement clinique, il faut quand même que tu aies un bagage de connaissances et d'expériences pour être capable de nuancer. Je pense que cela va déprendre du soutien de l'équipe qui va être en place, tu sais je veux dire peu importe la formation, peu importe où tu travailles, c'est la façon dont tu es coaché qui fait la différence. ( $P 2$, superviseur clinique).

L'équipe de superviseurs met en place des formations continues pour maintenir la qualité des interventions en ICI pour l'ensemble des intervenants afin d'harmoniser les connaissances tout comme un accompagnement individuel des intervenants selon leurs besoins. Ils peuvent aller observer les intervenants en situation d'intervention, offrir une supervision sous forme de discussion clinique et de conseils ou avoir recours à une auto-évaluation permettant que chacun des intervenants puissent s'évaluer et se fixer des objectifs de supervision. "Donc les intervenants vont s'auto-évaluer en début d'année. [...] À partir de là, ils vont se fixer des objectifs qu'on va les aider à travailler. » (P1) (superviseur)

En ce qui concerne les adaptations jugées favorables par les intervenants, ils rapportent que la supervision devrait être offerte de façon systématique et obligatoire et reposer sur des objectifs professionnels déterminés par les superviseurs et les intervenants eux-mêmes. Certains proposent même de faire une évaluation des connaissances de l'ICl chez les intervenants. "Tu sais de faire un test au début, pour que tout le monde ait les mêmes bases. » (P1). Ils souhaiteraient également une mise en place de rencontres d'échanges sur les pratiques régulières pour discuter des différents cas cliniques et des pratiques d'intervention.

Donc c'est cela, d'avoir quelque chose de plus uniforme et peutêtre de parler plus dans les réunions des interventions qu'on fait et de nos bons coups. Tu sais, peut-être de partir des thématiques, faire du co-développement, peut-être que cela pourrait être aidant. [...] Parce que là on travaille tous en sous-équipes, mais tu sais, qu'on mette tout cela ensemble. Parce que c'est tout le même programme au bout du compte. (P4)

\section{La participation}

4.1 La participation des parents. Cette dimension correspond à la participation des différents acteurs impliqués dans le programme. Selon les intervenants, la participation des parents représente une composante essentielle au programme $\mathrm{ICI}$. Ils rapportent que les parents devraient être impliqués dès le début de I'ICl pour le choix des objectifs d'intervention, ainsi que tout au long du 
programme pour la poursuite des interventions à domicile et la discussion entourant les stratégies d'intervention. "Ça fait partie de nos mandats, de transmettre aux parents ce qu'on fait. » (P7)

Ils proposent une adaptation au programme actuel et suggèrent la mise en place d'au moins une séance d'intervention par semaine en présence du parent afin de l'impliquer dans le processus d'intervention et de favoriser le transfert des informations concernant les objectifs et les stratégies d'intervention.

4.2 La participation des différents professionnels. Les intervenants soulignent qu'ils travaillent actuellement avec plusieurs partenaires tels que les écoles, les centres de réadaptation. Pour ce faire, divers moyens sont mis en œuvre. " On fait beaucoup de plans de services individualisés aussi. On essaie de s'arrimer. ॥ (P2). Dans certaines situations, la mise en place de cahiers de liaison est jugée favorable pour assurer les échanges réguliers entre les partenaires impliqués.

On a vraiment un cahier d'intervention et on le laisse dans les casiers. C'est vraiment écrit textuellement tous les bons coups, ce qu'on a travaillé et on essaie de donner des idées pour reprendre les choses à la maison. (P4)

De plus, les intervenants offrent de la formation aux acteurs externes au programme $\mathrm{ICl}$ pour faciliter les relations et l'arrimage des partenariats. Ils suggèrent de sensibiliser les différents milieux au programme $\mathrm{ICl}$ afin d'expliquer le rôle et les missions. "Je pense aussi que c'est de faire connaître qui on est, à quoi on sert. On n'est pas là pour te dire quoi faire, pour t'observer, pour te juger, puis souvent c'est l'impression qu'ils ont. »(P6)

Les intervenants soulignent que d'autres adaptations du programme seraient favorables à la participation car la collaboration est jugée inégale d'une situation à une autre. Ils estiment que l'ajout de mécanismes de collaboration formalisés par leur établissement, notamment, par la mise en place de plans de services et de rencontres d'échanges avec les milieux impliqués auprès de l'enfant serait favorable. Ils estiment également que les intervenants du programme $\mathrm{ICl}$ devraient offrir un soutien aux milieux externes qui accueillent un enfant présentant un TSA par le biais de conseils, de propositions de stratégies d'intervention et de conseils concernant l'adaptation des objectifs d'intervention ou de l'environnement.

\section{La différenciation}

Cette dimension concerne les caractéristiques uniques d'un programme qui le distingue des autres programmes habituellement implantés pour les enfants présentant un TSA. Les intervenants soulignent que le modèle préconisé par leur établissement les distingue des autres établissements qui implantent l'ICI par le choix qu'ils ont fait du modèle de l'ABA-VB et ce, pour tous les enfants du programme. Ils soulignent que le choix de modèle a plusieurs avantages et considèrent qu'il s'agit d'une valeur ajoutée par rapport à d'autres programmes $\mathrm{ICl}$ implantés dans différents services québécois. Ils insistent sur l'intérêt d'avoir opté pour un modèle 
d'intervention centré sur l'enseignement d'objectifs fonctionnels et ce, dans tous les milieux de vie de l'enfant et pour l'ensemble des sphères du développement.

On est vraiment dans tout ce qui est fonctionnel pour l'enfant dans sa réalité [...] On ratisse plus large, mais plus efficacement que juste d'apprendre à l'enfant ce qui ne lui servira pas. [...] Moi je pense qu'on est vraiment en train de créer un modèle. [...] On est dans la bonne voie. Je pense qu'on a un modèle fort, tu sais les intervenants, la force, ils portent un dossier au complet, ils voient tous les aspects et ce n'est pas quelqu'un qui leur dit applique tel programme comme ça peut se faire ailleurs. C'est une force, et ça demande beaucoup de soutien puis beaucoup de travail d'équipe.

(P2) (superviseur)

Enfin, les intervenants mentionnent que la formation initiale constitue un caractère unique dans leur établissement, car ceux-ci sont tous détenteurs d'une formation universitaire. «Quand le programme a été mis en place, la gestionnaire a vraiment insisté pour que ce soit des professionnels qui portent le dossier, par exemple, un psychoéducateur. » (P2)

Les intervenants soulignent que leur établissement se distingue par une prise de position claire sur un modèle d'intervention précis, soit l'ABA-VB. Ce choix permet un cadre clinique de référence entre les intervenants à partir d'un curriculum d'intervention qui permet à la fois d'évaluer le développement de l'enfant et de cibler des objectifs d'intervention fonctionnels dans les différents lieux de vie de l'enfant. Ils considèrent que le modèle préconisé dans leur établissement permet une intervention fonctionnelle ainsi qu'une préparation adéquate des enfants à intégrer le milieu scolaire.

\section{Discussion}

Dans le processus d'implantation des PBDP dans les services courants, certaines modifications peuvent être effectuées afin d'adapter les pratiques aux contextes dans lesquels elles s'implantent (Aarons et al., 2012; Escoffery et al, 2018; Pérez et al., 2016). Pour trouver l'équilibre entre la fidélité d'une PBDP et son implantation en contextes de services courants, la science de l'implantation recommande d'évaluer la fidélité de l'implantation pour vérifier comment les composantes essentielles d'une intervention sont implantées, mais aussi de documenter les adaptations effectuées en regard de l'intervention prévue à la base (Aarons et al., 2012; Chen, 2015; Dunsenbury et al., 2005; Escoffery et al, 2018; Pérez et al., 2016). En contexte québécois, I'ICl a été implanté à grande échelle dans les services depuis 2003. Les établissements ont effectué des choix variés et l'implantation de I'ICl ayant fait l'objet de plusieurs adaptations locales (Dionne et al., 2016; Gamache et al., 2010; Paquet et al., 2017). La présente étude visait à recueillir le point de vue des intervenants d'un de ces établissements concernant les composantes de leur programme ICI qu'ils implantent, ainsi que les adaptations qu'ils jugent favorables. 
D'abord, du point de vue de l'adhésion, les intervenants basent leur programme $\mathrm{ICl}$ sur le modèle ABA-VB et ils utilisent le curriculum VB-MAPP (Sundberg, 2008) comme outil de référence pour tous les enfants qui reçoivent l'ICI dans leur établissement. Ce choix d'offrir une intervention basée sur un curriculum de référence (Sundberg, 2008) diffère de ce qui est généralement rapporté par les représentants d'établissements québécois, optant plutôt pour diverses combinaisons de curriculums, tel que celui de Lovaas $(1987 ; 2003)$ ou Maurice, Green et Luce (1996) (Dionne et al., 2016). D'ailleurs, l'identification d'objectifs dans les différentes sphères de développement, dont celles qui sont particulièrement affectées par le TSA, sont considérées comme des caractéristiques essentielles de I'ICI (Perry et al., 2016).

Les composantes que les répondants jugent essentielles pour la mise en place de l'ICl sont : l'évaluation de l'enfant avec le curriculum VB-MAPP, l'évaluation des renforçateurs, la rédaction des objectifs d'intervention, la planification des stratégies spécifiques d'intervention, le recueil des données quotidienne, la révision des objectifs et la préparation de la transition vers le milieu scolaire. Ces composantes ont permis aux intervenants d'harmoniser les pratiques, notamment par la mise en place de documents de référence, tout en permettant d'individualiser l'intervention aux besoins de chacun des enfants et de mettre en œuvre l'intervention dans les différents milieux de vie. Ces composantes essentielles rejoignent celles qui ont été identifiées dans les écrits scientifiques, notamment, l'utilisation d'un curriculum d'intervention regroupant plusieurs domaines de développement déficitaires chez les enfants présentant un TSA, l'intervention dans les différents milieux de vie de l'enfant et la préparation à l'intégration au milieu scolaire (Perry et al., 2016). La méthode de recueil de données préconisée par les intervenants, soit celle par échantillonnage à froid (cold probe), comporte certains avantages, notamment celui du gain de temps (Cumming et Carr, 2009). Les avis concernant l'utilisation de la cotation en discontinu, tel que proposé, afin de remplacer les cotations en continu sont partagés, la cotation en discontinu comportant également d'importants avantages (p.ex., un suivi plus étroit des progrès de l'enfant, un monitorage plus étroit de l'intervention offerte) (Cumming et Carr, 2009; Giunta-Fede, Reeve, DeBar, Vladescu et Reeve, 2016; Eikeseth, 2010; Najdowski et al., 2009).

Concernant le dosage, les intervenants rapportent que le dosage actuel de I'ICl dans leur établissement correspond plutôt à 14 à 16 heures par semaine et non à une vingtaine d'heures tel que préconisé par le MSSS. Différentes raisons soutiennent cette adaptation du dosage, notamment, des raisons organisationnelles (ressources humaines) mais aussi cliniques et liées aux besoins des enfants. En ce sens, un dosage inférieur à la vingtaine d'heures peut être offert selon les besoins des enfants de leur service. Le défi demeure cependant de préciser le dosage idéal en fonction de ces caractéristiques individuelles de chacun des enfants. Ce dosage est inférieur au dosage recommandé de 20 à 40 heures par semaine (National Research Council, 2001; Perry et al., 2016) mais rejoint le dosage offert dans d'autres études québécoises (Dionne et al., 2017; Rivard et al., 2014).

En ce qui concerne la dimension de la qualité, les intervenants soulignent l'importance de créer une relation de confiance avec l'enfant tout au long de l'intervention pour maintenir la qualité de l'ICl. Ceci fait écho à d'autres écrits 
scientifiques qui ont évalué l'efficacité de la formation des intervenants à l'utilisation de la procédure du pairage avec l'enfant (pressession pairing) (Lugo et al. 2017). Celle-ci est considérée prometteuse pour réduire la nature aversive du contexte d'intervention, développer une relation positive entre l'enfant et l'intervenant et prévenir les troubles du comportement en séance. Elle peut se mettre en place dans divers contextes d'intervention par le biais de différentes stratégies telles que l'utilisation des activités et des jouets préférés de l'enfant (renforçateurs) et l'imitation des actions de celui-ci (Carbone, Morgenstern, Zecchin-Tirri et Kolbert, 2007; Kelly, Axe, Allen et Magire, 2015; Lugo, King, Lamphere et McArdle, 2017; McLauglin et Carr, 2005; Shillingsburg, Bowen et Shapiro, 2014).

Ils soulignent également que la mise en place de plusieurs piliers organisationnels est nécessaire pour assurer la qualité de I'ICl, notamment, la formation continue, le travail d'équipe, la supervision, l'auto-évaluation et les rencontres d'échanges sur les pratiques. La supervision clinique est aussi considérée comme étant un moyen d'assurer la qualité de I'ICl (Eikeseth, 2010; Eikeseth, Hayward, Gale, Gitlesen et Eldevik, 2009; Love, Carr, Almason et Petursdottir, 2009; Paquet et al., 2017). Par contre, le modèle de supervision adopté par cet établissement diffère des écrits scientifiques par son caractère non obligatoire ainsi que par sa fréquence variable et généralement inférieure à la fréquence hebdomadaire recommandée (Eikeseth, 2010).

Au plan de la participation, les intervenants mentionnent que celle des parents est essentielle, tout comme celle de plusieurs professionnels qui gravitent autour de l'enfant. Pour cela, ils estiment que l'intervention à domicile est favorable ainsi que la sensibilisation, la formation des intervenants externes au programme ICl et la mise en place de mécanismes formels de collaboration. Ce choix permet une meilleure adhésion des parents à l'intervention tout en favorisant le développement des compétences des parents dans la gestion des comportements défis tout comme dans la poursuite de l'intervention au domicile familial. Ce choix rejoint les écrits scientifiques soulignant l'importance de l'implication parentale dans l'intervention (INESSS, 2014), tout comme celle des différents acteurs impliqués auprès de l'enfant (Perry et al., 2016).

Enfin, concernant la différenciation, la composante essentielle identifiée par les intervenants est l'embauche d'intervenants détenteurs d'une formation universitaire, dont plusieurs issus de la psychoéducation. Ce choix a été effectué par les gestionnaires du programme afin que les intervenants puissent être autonomes et responsables dans leur charge de cas, sans devoir avoir recours au superviseur pour la prise de décision clinique concernant l'orientation du programme de l'enfant. Bien que ceux-ci ne possèdent pas la certification au Behavior Analyst Certification Board (BACB), les intervenants interrogés semblent intégrer au programme ICI les compétences-clés identifiées dans les écrits scientifiques telles que l'évaluation fonctionnelle, les procédures de renforcement et les principes d'apprentissage (Eikeseth et al. 2009). L'intervention tout comme la supervision sont offertes par des cliniciens détenteurs d'une formation universitaire, dans la majorité des cas des psychoéducateurs possédant une expérience clinique en $\mathrm{ICl}$, ce qui est comparable aux données d'autres études (Love et al., 2009; Paquet et al. 2017). 
L'établissement québécois concerné par cette étude semble avoir réussi à trouver un équilibre entre la fidélité et l'adaptation du programme $\mathrm{ICI}$ en mettant en œuvre la majorité des composantes essentielles identifiées dans les écrits scientifiques sur l'ICl, telles que le groupe d'âge de référence, l'utilisation des stratégies d'enseignement basées sur l'AAC, la collecte de données; l'implication des parents, la généralisation des habiletés dans les différents milieux de vie, l'intégration en milieu ordinaire ainsi que la préparation au milieu scolaire (Eikeseth, 2009; Eikeseth, 2017; National Research Council [NRC], 2001; Perry, 2002; Perry et al. 2016; Rogers et Vismara, 2008). L'utilisation du modèle ABA-VB et du curriculum VB-MAPP permet une intervention globale en intégrant plusieurs sphères du développement dans I'ICI (la communication, l'imitation, la perception visuelle et l'appariement, le jeu, le comportement social, la routine de classe et les habiletés de groupe, la lecture, l'écriture et les mathématiques), tout comme la réduction des comportements-défis (Sundberg, 2008; Dixon, Peach et Daar, 2017).

Par contre, nonobstant les lignes directrices offertes par le MSSS, les intervenants interrogés perçoivent que diverses adaptations sont favorables pour la mise en place de l'ICl dans leur établissement. Les principales adaptations effectuées sont conformes à celles qui sont généralement effectuées dans les milieux de pratique, notamment, l'ajout de matériel pédagogique, la modification du dosage de l'intervention et l'intégration de nouvelles approches d'intervention, mais aucune modification n'a été effectuée en ce qui concerne le public-cible (Escoffery et al., 2018). L'établissement concerné par cette étude semble se distinguer des établissements québécois par une position claire concernant un modèle clinique de référence (Dionne et al., 2016; Gamache et al., 2010), soit l'ABA-VB, ce qui semble favoriser l'harmonisation de l'ICI d'un intervenant à un autre.

\section{Conclusion}

La présente étude visait à recueillir le point de vue des intervenants en ce qui concerne les composantes essentielles du programme ICl ainsi que les adaptations jugées favorables. Le modèle de Dane et Schneider (1998) utilisé a permis de recueillir une multitude d'informations sur le programme selon cinq dimensions, soit l'adhésion, le dosage, la qualité, la participation et la différenciation. Les propos de 10 intervenants qui appliquent I'ICl ont été recueillis à partir d'entretien semidirigés puis analysés à partir des dimensions d'un modèle d'évaluation de la fidélité de l'intervention. Les résultats obtenus illustrent la perception des intervenants qui appliquent l'ICl sur le terrain, ce qui constitue la force de cette étude. Leurs points de vue ont permis de contextualiser l'implantation de l'ICl dans un contexte de services courants et d'examiner les adaptations effectuées. Ces résultats représentent, bien entendu, le contexte singulier d'un établissement à un moment précis et les propos recueillis témoignent des perceptions des intervenants d'un contexte spécifique. Les propos analysés portent sur la perception des intervenants interrogés, ils sont donc teintés par leur subjectivité tout comme leur contexte singulier. Les résultats ne sont pas transférables à d'autres établissements québécois. Malgré leur apport, l'utilisation des données auto-rapportées peut présenter certains biais liés à un désir des cliniciens de fournir des informations positives sur l'adhésion au programme (Breitenstein et al. 2010; Perepletchikova, Treat et Kazdin, 2007). 
De plus, les dimensions abordées sont celles qui sont suggérées par le modèle d'évaluation de la fidélité d'implantation de Dane et Schneider (1998). Bien que ce modèle permette de considérer un ensemble de facteurs utiles pour documenter la façon dont est implanté un programme, il n'a pas été conçu dans la perspective de différencier spécifiquement les composantes essentielles. Aussi, il faut souligner que les différentes composantes du modèle (adhésion, qualité, dosage, participation) peuvent parfois se chevaucher, rendant difficile le classement de certains éléments liés à l'implantation du programme ( Carroll et al. 2007). II est également possible que l'utilisation d'un autre modèle d'évaluation ait pu mettre en exergue d'autres aspects du programme ou encore une catégorisation différente. Enfin, cette étude n'aborde pas la perception des parents ni celle des acteurs qui gravitent autour de I'ICI par rapport aux composantes essentielles, ce qui pourrait être pertinent afin de compléter le portrait et apporter certaines nuances.

Étant donné l'augmentation importante des diagnostics de TSA, les services publics ont une responsabilité d'avoir recours aux PBDP. Cependant, leur implantation dans les services publics courants ne va pas de soi et des mécanismes devraient être mis en place pour assurer le lien entre les connaissances scientifiques et la pratique. L'évaluation de la fidélité constitue l'un de ces moyens. Pour ce faire, la définition des composantes essentielles de l'intervention est nécessaire afin de trouver l'équilibre entre la fidélité et l'adaptation des pratiques dans le contexte de services courants. Le savoir issu de la pratique (bottom-up) combiné au savoir scientifique (top-down) permet de réduire l'écart entre les connaissances scientifiques et les services d'intervention courants et de mieux connaitre comment une PBDP s'implante dans le contexte réel (Bania, Roebuck et Chase, 2017; Chen, 2010).

À ce jour, les orientations fournies aux établissements responsables de l'implantation de l'ICl ne semblent pas assez précises pour assurer une implantation fidèle et uniforme de ce programme provincial, car elles balisent certains éléments tels que le dosage, mais laissent une marge d'interprétation ou " d'adaptation » importante. II serait donc souhaitable que les établissements puissent avoir accès à de la documentation rendant explicite les composantes essentielles du programme ainsi qu'à un outil d'évaluation de la fidélité avec laquelle le programme est mis en place. De plus, l'évaluation continue de la fidélité de l'ICI devrait constituer une règle et non une exception afin de prévenir les « adaptations » risquant d'amenuiser les effets du programme, tout en laissant une marge de manœuvre aux établissements. Dans les situations "d'adaptation » du programme ICl, celles-ci devraient être planifiées rigoureusement et faire l'objet d'évaluation continue. Bien que le dosage soit l'une des variables de l'évaluation de la fidélité, cette composante n'est pas suffisante pour juger de l'implantation optimale d'un programme. En ce sens, dans le contexte québécois, l'évaluation du dosage ne devrait pas constituer la seule cible d'évaluation, car elle n'est pas garante de la fidélité ni de la qualité de l'ICl.

À la lumière des propos recueillis dans le cadre de cette étude et des connaissances scientifiques, il apparaît souhaitable de s'intéresser davantage à la question de la mise en place des composantes essentielles de la fidélité de I'ICl mais aussi aux adaptations de ce programme implanté en contextes de services courants. Cela permettrait de fournir des clarifications aux établissements 
concernés par l'implantation et d'assurer une harmonisation des pratiques offertes aux jeunes enfants ayant un TSA dans les services québécois. Pour ce faire, il serait également intéressant de poursuivre les démarches d'identification des composantes essentielles de I'ICI. Cela permettrait, au plan de l'évaluation de l'implantation, de construire des devis d'évaluation de la fidélité qui considèrent les caractéristiques spécifiques des modèles de même que les caractéristiques communes entre les différents programmes. Cela serait utile également dans les études d'évaluation des effets, en considérant l'implantation des programmes et en facilitant les comparaisons entre les différents programmes. Aussi, de telles démarches contribueraient, dans un contexte de ressources limitées, à identifier les composantes pour lesquelles aucune économie ne doit être faite.

\section{Références}

Aarons, G. A. et Palinkas, L. A. (2007). Implementation of evidence based practice in child welfare: Service provider perspectives. Administration and Policy in Mental Health and Mental Health Services Research, 34(4), 411419. doi: $10.1007 / \mathrm{s} 10488-007-0121-3$

Aarons, G. A., Green, A. E., Palinkas, L. A., Self-Brown, S., Whitaker, D. J., Lutzker, J. R. et Chaffin, M. J. (2012). Dynamic adaptation process to implement an evidence-based child maltreatment intervention. Implementation Science, 7(32), 1-9. doi:10.1186/1748-5908-7-32

Agence de la santé publique du Canada (2018). Trouble du Spectre de l'autisme chez les enfants et les adolescents au Canada 2018. Un Rapport Du Système National de Surveillance du Trouble Du Spectre De l'autisme. Ottawa : Agence de la santé publique du Canada. Retiré de : https://www.canada.ca/content/dam/ phac-aspc/documents/services/publica tions/diseases-conditions/autism-spectr um-disorder-children-youth-canada2018/trouble-spectre-autisme-enfantsadolescents-canada-2018.pdf

Backer, T. E. (2001). Finding the balance: Program fidelity and adaptation in substance abuse prevention: A Stateof-the-art Review. Rockville, MD: Department of Health and Human Services, Substance Abuse and Mental Health Services Administration. Repéré à : https://www.csun.edu/sites/default/ files/FindingBalance1.pdf
Bania, M., Roebuck, B. et Chase, V. (2017). Local adaptations of crime prevention programs: Finding the optimal balance between fidelity and fit - A Literature Review. Ottawa, ON: Public Safety Canada. (Research Report 2017-R019). doi: 10.13140/RG.2.2.20315.41767

Bérubé A., Lafantaisie, V., Dubeau, D., Coutu, S., Caron, J. et Devault, A. (2014). Using implementation evaluation to uncover a child neglect prevention program. Evaluation and Program Planning, 45, 1-8. doi: 10.1016/j. evalprogplan.2014.03.001

Bondy, A. et Frost, L. (1994). The picture exchange communication system. Focus on Autistic Behavior, 9(3), 1-19. doi: $10.1177 / 108835769400900301$

Breitenstein, S., M., Gross, D., Garvey, C., Hill, C., Fogg, L. et Resnick, B. (2010). Implementation fidelity in communitybased interventions. Research in Nursing and Health, 33(2), 164-173. doi:10.1002/nur.20373

Bryson, S. E., Koegel, L. K., Koegel, R. L. et Openden, D. (2007). Large scale dissemination and community implementation of pivotal response treatment: Program description and preliminary data. Research \& Practice for Persons with Severe Disabilities 32(2), 142-153. doi: 10.2511/rpsd.32.2.142 
Carbone, V. J., Morgenstern, B., ZecchinTirri, G. et Kolberg, L. (2007). The role of the reflexive conditioned motivating operation (CMO-R) during discrete trial instruction of children with autism. Journal of Early Intensive Behavioral Intervention, 4(4), 658-680. doi: 10.1037/h0100399

Caron, V., Bérubé, A. et Paquet, A. (2017). Implementation evaluation of early intensive behavioral intervention programs for children with autism spectrum disorders: A systematic review of studies in the last decade. Evaluation and Program Planning, 62, 1-8. doi: 10.1016/j.evalprogplan.2017.01.004

Carroll, C., Patterson, M., Wood, S., Booth, A., Rick, J. et Balain, S. (2007) A conceptual framework for implementation fidelity. Implementation Science, 2(40), 1-9. doi. org/10.1186/1748-5908-2-40

Castro, F. G., Barrera, M. et Martinez, C. R. (2004). The cultural adaptation of prevention interventions: Resolving tensions between fidelity and fit. Prevention Science, 5(1), 41-45. doi.org/10.1023 /B:PREV.0000013980.12412.cd

Chen, H. T. (2010). Bottom-up approach to integrative validity: A new perspective for program evaluation. Evaluation and Program Planning, 33, 205-214. doi: 10.1016/j.evalprogplan.2009.10.002

Chen, H. T. (2015). Practical Program Evaluation. Theory-driven evaluation and the integrated evaluation perspective, ( $2^{\mathrm{e}}$ éd). Los Angeles, CA: Sage Publications.

Cook, B. G. et Odom, S. L. (2013). Evidencebased practices and implementation science in special education. Exceptional Children, 79(2), 135-144. doi: 10.1177/001440291307900201

Crooke, P. J. et Oslwang, L. B. (2015). Practice-based research: another pathway for closing the research-practice gap, Journal of Speech, Language, and Hearing Research, 58, 1871-1882. doi: 10.1044/2015_JSLHR-L-15-0243.
Cummings, A. R. et Carr, J. E. (2009). Evaluating progress in behavioral programs for children with autism spectrum disorders via continuous and discontinuous measurement. Journal of Applied Behavior Analysis, 42, 57 -71. doi:10.1901/jaba.2009.42-57.

Dane, A. V. et Schneider, B. H. (1998). Program integrity in primary and early secondary prevention: Are implementation effects out of control? Clinical Psychology Review, 18(1), 2345. doi: 10.1016/S0272-7358(97)000433

Dionne, C., Joly, J., Paquet, A., Rousseau, M. et Rivard, M. (2016). Organizations' choices when implementing an early intensive behavioral intervention program (EIBI). Education Sciences et Society, 2, 150-170. doi: 10.3280/ess2$20160 a 3934$

Dixon, M. R., Peach, J. et Daar, J. H. (2017). Teaching complex verbal operants to children with autism and establishing generalization using the Peak curriculum, Journal of Applied Behavior Analysis, 50(2), 317-331.

Dunsenbury, L., Brannigan, R., Falco, M. et Hansen, W. B. (2003). A review of research on fidelity of implementation: Implications for drug abuse prevention in school settings. Health Education Research, 18(2), 237-256. doi: 10.1093/ her/18.2.237

Dunsenbury, L., Brannigan, R., Hansen, W. B., Walsh, J. et Falco, M. (2005). Quality of implementation: developing measures crucial to understanding the diffusion of preventive interventions. Health Education Research: Theory \& Practice, 20(3), 308-313. doi: 10.1093/ her/cyg134

Durlak, J. A. et Dupré, E. P. (2008). Implementation matters: A review of research on the influence of implementation on program outcomes and the factors affecting implementation. American Journal of Community Psychology, 41, 327-350. doi: 10.1007/ s10464-008-9165-0 
Eikeseth, S. (2009). Outcome of comprehensive psycho-educational interventions for young children with autism. Research in Developmental Disabilities, 30(1), 158-78. doi: 10.1016/j.ridd.2008.02.003.

Eikeseth, S. (2010). Examination of qualifications required of an EIBI professional. European Journal of Behavior Analysis, 11(2), 239-246.

Eikeseth, S., Hayward, D., Gale, C., Gitlesen, J.-P. et Eldevik, S. (2009). Intensity of supervision and outcome for preschool aged children receiving early and intensive behavioral interventions: $A$ preliminary study. Research in Autism Spectrum Disorders, 3, 67-73. doi: 10.1016/j.rasd.2008.04.003

Eikeseth, S. (2017). Additional treatment parameters and issues requiring study: Early intensive behavioral intervention (EIBI). Dans J. Matson (dir.), Handbook of Treatments for Autism Spectrum Disorder. Autism and Child Psychopathology Series. (p. 209-230). Bâle, Suisse: Springer International Publishing AG.

Eikeseth, S., Klintwall, L., Jahr, E. et Karlsson, P. (2012). Outcome for children with autism receiving early and intensive behavioral intervention in mainstream preschool and kindergarten settings. Research in Autism Spectrum Disorders, 6, 829-835. doi: 10.1016/j. rasd.2011.09.002

Eldevik, S., Hastings, R. P., Hughes, J. C., Jahr, E., Eikeseth, S. et Cross, S. (2009). Meta-analysis of early intensive behavioral intervention for children with autism. Journal of Clinical Child and Adolescent Psychology, 38, 439-450. doi: 10.1080/15374410902851739

Escoffrey, C, Lebow-Skelley, E., Haardoefer, R., Boing, E., Udelson, H., Wood, R. et al. (2018). A systematic review of adaptations of evidence-based public health interventions globally, Implementation Science, 13(125), 1-21. doi: 10.1186/s13012-018-0815-9

Fixsen, D., Blase, K., Metz, A. et Van Dyke, M. (2013). Statewide implementation of
evidence-Based Programs. Exceptional Children, 79(2), 213-230. doi: 10.1177/001440291307900206

Fixsen, D. L., Naoom, S. F., Blase, K. A., Friedman, R. M. et Wallace, F. (2005). Implementation Research: A synthesis of the literature (FMHI \#231). Tampa, FL: University of South Florida, Louis de la Parte Florida Mental Health Institute, The National Implementation Research Network. Repéré à: https://fpg.unc.edu/ node $/ 4445$

Gamache, V., Joly, J. et Dionne, C. (2010). La fidélité et qualité d'implantation du programme québécois d'intervention comportementale intensive destiné aux enfants présentant un trouble envahissant du développement en CRDITED. Revue de psychoéducation, 40(1), 1-23.

Giunta-Fede, T. A., Reeve, S. A., DeBar, R. M., Vladescu, J. C., et Reeve, K. F. (2016). Comparing continuous and discontinuous data collection during discrete trial teaching of tacting by children with autism, Behavioral Interventions, 31, 311-331. doi: 10.1002/bin.1446

Gray, C., Broek, E., Cain, S. L., Dutkiewicz, M., Fleck, C., Gray, B., Gray, J., Jonker, S., Lindrup, A. et Moore, L. (dir.). (1993). The social story book. Jenison, MI: Jenison Public Schools.

Gresham, F. M., Gansle, K. A. et Noell, G. H. (1993). Treatment integrity in applied behavior analysis with children, Journal of Applied Behavior Analysis, 26, 257263. doi:10.1901/jaba.1993.26-257

Hagermoser Sanetti, L. M. et Kratchowill, T. R. (2009) Toward developing a science of treatment integrity: Introduction to the special series, School Psychology Review, 38(4), 445-459.

Hansen, W. B., Pankratz, M. M., Dusenbury, L., Giles, S. M., Bishop, D. C., Albritton, J. et Strack, J. (2013). Styles of adaptation: The impact of frequency and valence of adaptation on preventing substance use. Health Education, 113, 345-363. doi:10.1108/09654281311329268 
Huffman, L. C., Sutcliffe, T. K., Tanner, I. S. D. et Feldman, H. M. (2011). Management of symptoms in children with autism spectrum disorders: A comprehensive review of pharmacologic and complementary-alternative medicine treatments. Journal of Developmental and Behavioral Pediatrics, 32, 56-68.

Institut national d'excellence en santé et en services sociaux (INESSS) (2014a). L'efficacité des interventions de réadaptation et des traitements pharmacologiques pour les enfants de 2 à 12 ans ayant un trouble du spectre de l'autisme (TSA): édition revisée. ETMIS, 9(6), 1-67. Repéré à: https://www.inesss. qc.ca/fileadmin/doc/INESSS/Rapports/ ServicesSociaux/INESSS_Interventions Readap_TraitementPharmaco_Enfants Aut.pdf.

Institut national d'excellence en santé et en services sociaux (INESSS). (2014b). Addenda. L'efficacité des interventions de réadaptation et des traitements pharmacologiques pour les enfants de 2 à 12 ans ayant un trouble du spectre de l'autisme (TSA). Repéré à https://www. inesss.qc.ca/fileadmin/doc/INESSS/ Rapports/ServicesSociaux/INESSS Addenda _ TSA.pdf

Institut National de Santé publique du Québec (2017). Surveillance du trouble du spectre de l'autisme au Québec. Repéré à: https://www.inspq.qc.ca/sites/default/ files/publications/2310_surveillance_ trouble_spectre_autisme.pdf

Kelly, A. N., Axe, J. B., Allen, R. F. et Maguire, R. W. (2015). Effect of presession pairing on the challenging behavior and academic responding of children with autism. Behavioral Interventions, 30, 135-156. doi: 10.1002/bin.1408

Leclair, I.-A., Paquette, G. et Letarte, M.J. (2017). La fidélité d'un programme probant au-delà de son implantation initiale : l'exemple de Ces années incroyables en protection de l'enfance de 2003 à 2013. Canadian Journal of Program Evaluation, 32(1), 90-108. doi: 10.3138/cjpe.31142
Lindstead, E., Dixon, D. R., Hong, E., Burns, C. O., French, R., Novack, M. N. et Granspeesheh, D. (2017). An evaluation of the effects of intensity and duration on outcomes across treatment domains for children with autism spectrum disorder, Translational Psychiatry, 7, e1234. doi: 10.1038/tp.2017.207

Lee, S. J., Altschul, A. I. et Mowbray, C. T. (2008). Using planned adaptation to implement evidence-based programs with new populations. American Journal on Community Psychology, 41, 290-303. doi: $10.1007 / \mathrm{s} 10464-008-9160-5$

Lovaas O.I. (1987). Behavioral treatment and normal educational and intellectual functioning in young autistic children. Journal of Consulting and Clinical Psychology, 55, 3-9. DOI: 10.1037/0022006X.55.1.3

Lovaas O.I. (2003). Teaching Individuals with Developmental Delays: Basic Intervention Techniques. Austin, TX: Pro-Ed.

Love J. R., Carr J. E., Almason S. M. and Petursdottir A. I. (2009). Early and intensive behavioral intervention for autism: A survey of clinical practices. Research in Autism Spectrum Disorders, 3, 421-428. doi: 10.1016/j. rasd.2008.08.008.

Lugo, A. M., King, M. L., Lamphere, J. C. et McArdle, P. E. (2017). Developing procedures to improve therapist-child rapport in early intervention, Behavior Analysis in Practice, 10(4), 395-401. doi 10.1007/s40617-016-0165-5

Makrygianni, M. K., Gena, A., Katoudi, S., Galanis, P. (2018). The effectiveness of applied behavior analytic interventions for children with Autism Spectrum Disorder: A meta-analytic study. Research in Autism Spectrum Disorders, 5, 18-31. doi: 10.1016/j.rasd.2018.03.006

Makrygianni, M. K. et Reed, P. (2010). A metaanalytic review of the effectiveness of behavioral early intervention programs for children with autistic spectrum disorders. Research in Autism Spectrum Disorders, 4, 577-593. doi: 10.1016/j. rasd.2010.01.014 
Maurice C., Green G. and Luce S. (1996). Behavioral intervention for young children with autism: A manual for parents and professionals. Austin, TX: Pro-Ed.

Mclntyre, L. L., Gresham, F. M., DiGennaro, F. D. et Reed, D. D. (2007). Treatment integrity of school-based interventions with children in the Journal of Applied Behavior Analysis 1991-2005. Journal of Applied Behavioral Analysis, 40, 659972. doi: 10.1901/jaba.2007.659-672

McLaughlin, D. M. et Carr, E. G. (2005). Quality of rapport as a setting event for problem behavior: assessment and intervention. Journal of Positive Behavior Interventions, 7(2), 68-91. doi: $10.1177 / 10983007050070020401$

Metz, A., Halle, T., Bartley, L. et Blasberg, A. (2013). The key components of successful implementation. Dans T. Halle, A. Metz, et I. Martinez-Beck (dir.), Applying implementation science in early childhood programs and systems (pp. 21-42). Baltimore, MD, US: Paul H Brookes Publishing.

Michie, S., Fixsen, D., Grimshaw, J. M. et Eccles, M. P. (2009). Specifying and reporting complex behaviour change interventions: the need for a scientific method. Implementation Science, 4(40), 1-6. doi: 10.1186/1748-5908-4-40

Ministère de la Santé et des Services sociaux. (2003). Un geste porteur d'avenir - Des services aux personnes présentant un trouble envahissant $d u$ développement, à leurs familles et à leurs proches. Québec: Ministère de la Santé et des Services sociaux. Repéré à : http://publications.msss.gouv.qc.ca/ msss/document-000837/

Ministère de la Santé et des Services sociaux. (2017). Des actions structurantes pour les personnes et leur famille. Plan d'action sur le trouble du spectre de l'autisme 2017-2022. Québec : Ministère de la Santé et des Services sociaux. Repéré à : http://publications. msss.gouv.qc.ca/msss/fichiers/2016/16824-06W.pdf

Moncher, F. J. et Prinz, R. J. (1991) Treatment fidelity in outcome studies. Clinical
Psychology Review, 11, 247-266. doi: 10.1016/0272-7358(91)90103-2

Najdowski, A. C., Chilingaryan, V., Bergstrom, R., Granpeesheh, D., Balasanyan, S., Aguilar, B. et Tarbox, J. (2009). Comparison of data-collection methods in a behavioral intervention program for children with pervasive developmental disorders: A replication. Journal of Applied Behavior Analysis, 42, 827-832. doi: 10.1901/jaba.2009.42-827

National Autism Center. (2009). National standards report. The national standards project addressing the need for evidence-based practice guidelines for autism spectrum disorders. Randolph, MA: National Autism Center. Repéré à: dhttp://www.nationalautismcenter.org/ national-standards-project/

National Autism Center. (2015). Findings and conclusions: National standards project, phase 2. Randolph, MA: National Autism Center. Retiré de: http:// www.nationalautismcenter.org/nationalstandards-project/phase-2/

National Research Council. (2001). Educating children with autism (Committee on Educational Interventions for Children with Autism, Division of Behavioral and Social Sciences and Education). Washington, DC: National Academy Press.

Odom, S. L., Boyd, B. A., Hall, L. J. et Hume, K. (2010). Evaluation of comprehensive treatment models for individuals with autism spectrum disorders. Journal of Autism and Developmental Disorders, 40, 425-436. doi: 10.1007/s10803-0090825-1

Odom, S. L., Collet-Klingenberg, L., Rogers, S. J. et Hatton, D. D. (2010). Evidencebased practices in interventions for children and youth with autism spectrum disorders. Preventing School Failure, 54(4), 275-282. doi: 10.1080/10459881003785506

Odom, S. L., Cox, A.W. et Brock, M. E. (2013). Implementation science, professional development, and autism spectrum disorders. Exceptional Children, 79(2), 233-251. doi: 10.1177/001440291307900207 
Olswang, L. B. et Prelock, P. A. (2015). Bridging the gap between research and practice: implementation science, Journal of Speech, Language, and Hearing Research, 58, 1818-1826. doi: 10.1044/2015_JSLHR-L-14-0305

Paillé, P. et Mucchielli, A. (2012). L'analyse qualitative en sciences humaines et sociales ( $3^{e}$ éd.). Paris, France : Armand Colin.

Paquet, A., Dionne, C., Joly, J., Rousseau, M., et Rivard, M. (2017). Supervision of large-scale community-based early intensive behavioural intervention programs in Quebec: Description of practices. Journal on Developmental Disabilities, 23(1), 54-63. Repéré à: $\quad$ https://oadd.org/wp-content/ uploads/2017/11/41024-JoDD-23-1-v9f54-63-Paquet-et-al-1.pdf

Paquette, G., Joly, J. et Tourigny, M. (2010). La fidélité de l'implantation d'un programme d'intervention pour des adolescentes agressées sexuellement: une mesure par observation systématique. Revue canadienne d'évaluation de programme, 24(2), 5779. Repéré à: https://evaluationcanada. $\mathrm{ca} / \mathrm{fr} / \mathrm{la}$-fidelite-de-limplantation-dunprogramme-dintervention-pour-desadolescentes-agressees

Perepletchikova, F., Treat, T. A, Kazdin, A. E. (2007). Treatment integrity in psychotherapy research: Analysis of the studies and examination of the associated factors. Journal of Consulting \& Clinical Psychology, 75(6), 829-841. doi:10.1037/0022-006X.75.6.829

Pérez, D., Van der Stuyft, P. V., Carmen Zabala, M., Castro, M. et Lefèvre, P. (2016). A modified theoretical framework to assess implementation fidelity of adaptive public health interventions. Implementation Science, 11(91), 1-11. doi: 10.1186/s13012-016-0457-8.

Perry, A. (2002). Intensive early intervention program for children with autism: Background and design of the Ontario preschool autism initiative. Journal on Developmental Disabilities, 9(2), 121128. Repéré à: https://oadd.org/wpcontent/uploads/2016/12/art11Perry.pdf
Perry, A., Cummings, A., Dunn Geier, J., Freeman, N., Hughes, S., LaRose, L., et al. (2008). Effectiveness of intensive behavioral intervention in a large, community-based program. Research in Autism Spectrum Disorders, 2, 621642. doi:10.1016/j.rasd.2011.09.011

Perry, A., Koudys, J. et Blacklock, K. (2016). Early intensive behavioral intervention. Dans N. N. Singh (dir.), Handbook of Evidence-Based Practices in Intellectual Developmental Disabilities, EvidenceBased Practices in Behavioral Health. (p. 511-535). Bâle, Suisse: Springer International Publishing.

Peterson, L., Horner, A. L. et Wonderlich, S. A. (1982). The integrity of independent variables in behavior analysis. Journal of Applied Behavioral Analysis, 15(4), 477-492. doi:10.1901/jaba.1982.15-477

Reichow, B. (2012). Overview of metaanalyses on early intensive behavioral intervention for young children with autism spectrum disorders. Journal of Autism and Developmental Disorders, 42(4), 512-520. doi: 10.1007/s10803011-1218-9

Reichow, B., Barton, E. E, Boyd, B. A. et Hume, K. (2012). Early Intensive Behavioral Intervention (EIBI) for Young Children with Autism Spectrum Disorders (ASD): A Systematic Review. Cochrane Database Syst Rev, 17(10), doi: $\quad 10.1002 / 14651858 . C D 009260$. pub2.

Reichow, B., Hume, K., Barton, E. E. et Boyd, B. A. (2018). Early intensive behavioral intervention (EIBI) for young children with autism spectrum disorders (ASD). Cochrane Database of Systematic Reviews, Issue 5, Art. No.: CD009260. https://doi: 10.1002/14651858.CD00926 0. pub3

Rivard, M., Therroux, A. et Mercier, C. (2014). Effectiveness of early intensive behavioral intervention in public and mainstream setting: The case of preschool age children with autism spectrum disorders. Research in Autism Spectrum Disorders, 8, 1031-1043. DOI: 10.1016/j.rasd.2014.05.010 
Rogers, S. J. et Vismara, L. A. (2008). Evidence-based comprehensive treatments for early autism. Journal of Clinical Child and Adolescent Psychology, 37, 8-38. doi: 10.1080/15374410701817808

Royer, C., Baribeau, C. et Duschesne, A. (2009). Les entretiens individuels dans la recherche en sciences sociales au Québec : où en sommes-nous? Un panorama des usages. Recherches qualitatives, Hors Série, 7, 64-79. Retiré de: http://www.recherche-qualitative. qc.ca/documents/files/revue/hors_serie/ hors_serie_v7/HS7_Texte_Royer_ Baribeau.pdf

Shillingsburg, M. A., Bowen, C. N. et Shapiro, S. K. (2014). Increasing social approach and decreasing social avoidance in children with autism spectrum disorder during discrete trial training. Research in Autism Spectrum Disorders, 8(11), 1443-1453. doi: 10.1016/j. rasd.2014.07.013

Skinner, B.F. (1957). Verbal Behavior. New York: Prentice Hall.
Sundberg, M. L. (2008). The verbal behavior milestones assessment and placement program: The VB-MAPP. Concord, CA: AVB Press.

Stock, R., Mirenda, P. et Smith, I. (2013). Comparison of community-based verbal behavior and pivotal response treatment for young children with autism spectrum disorders. Research in Autism Spectrum Disorders, 7, 1168-1181. doi 10.1016/j. rasd.2013.06.002

Virués-Ortega, J. (2010). Applied behavior analytic intervention for autism in early childhood: meta-analysis, metaregression and dose-response metaanalysis of multiple outcomes. Clinical Psychology Review, 4, 387-99. doi: 10.1016/j.cpr.2010.01.008

Vivanti, G., Kasari, C., Green, J., Mandell, D., Maye, M. et Hudry, K. (2018). Implementing and evaluating early intervention for children with autism: Where are the gaps and what should we do? Autism Research, 11(1), 16-23. doi: 10.1002/aur.1900 